\title{
Die entwicklungspsychologische Sprachtherapie mit kleinen Kindern
}

\section{The Developmental Language Therapy with Small Children}

\author{
Autor \\ B. Zollinger \\ Institut \\ Zentrum für kleine Kinder GmbH, Winterthur, Schweiz
}

Schlüsselwörter
Individuation
Repräsentation
Sprachverstehen
frühe Spracherwerbs-
störungen
Frühtherapie
Key words
individuation
representation
language comprehension
late talkers
early intervention

Bibliografie

DOI http://dx.doi.org/ 10.1055/s-0034-1395631 Sprache · Stimme · Gehör 2014; 38: 163-166 (c) Georg Thieme Verlag KG Stuttgart · New York ISSN 0342-0477

Korrespondenzadresse Dr. phil. Barbara Zollinger Zentrum für kleine Kinder $\mathrm{GmbH}$ Pionierstraße 10 8400 Winterthur Schweiz zollinger@kinder.ch

\section{Zusammenfassung \\ $\nabla$}

Einleitend legt die Autorin die theoretischen Zusammenhänge dar, auf denen die entwicklungspsychologische Sprachtherapie basiert. Im Mittelpunkt stehen dabei

1. die Erfahrung der Selbstwirksamkeit als wichtiger Bestandteil der Individuationsprozesse und somit der kommunikativen Funktion der Sprache,

2. das Handlungsresultat und seine Bedeutung als Grundlage für die symbolische Entwicklung und die repräsentative Funktion der Sprache, und

3. der trianguläre Blickkontakt als Basis für den Aufbau des Sprachverstehens und einer aktiven Spracherwerbsstrategie.

\section{Abstract \\ $\nabla$}

As an introduction, the author lays out the theoretical coherences upon which the developmental language therapy is based. Points of focus are thereby

1. the experience of self-efficacy as an important part of the individuation processes and so the communicative function of language,

2. the experience of transformation by action as a prerequisite of symbolic development and so the representative function of language, and

3. the triangular eye contact as a base for language comprehension and so an active language acquisition strategy.

\section{Lernziel}

Da der vorgestellte Therapieansatz stark entwicklungspsychologisch orientiert ist, sollen die Leserinnen und Leser wissen, welche Prozesse den Spracherwerb und eine mögliche Dynamik früher Spracherwerbsstörungen bestimmen. Im 2. Teil des Artikels sollen sie dann die Grundhaltung sowie die wichtigsten Elemente der entwicklungspsychologischen Sprachtherapie mit kleinen Kindern kennenlernen.

\section{Einleitung \\ $\nabla$ \\ Entwicklung der Individuation}

Sprache hat 2 Hauptfunktionen: einerseits die Kommunikation mit dem Ziel, die Welt zu verändern, und andererseits die Repräsentation mit dem Ziel, die Welt darzustellen.

Die Voraussetzung für die Entwicklung der kommunikativen Funktion liegt im Anspruch, anderen Personen etwas mitzuteilen (Sprachproduktion), bzw. den Äußerungen anderer Personen zuzuhören (Sprachverstehen). Die Prozesse, welche zu diesen Kompetenzen führen, geschehen in der Auseinandersetzung mit der Personenwelt (Ich - Du) und können durch die Entwicklung der Individuation umschrieben werden [1].

\section{Auseinandersetzung mit der Gegenstandswelt}

Die Voraussetzung für die Entwicklung der repräsentativen Funktion von Sprache liegt als erstes darin, verlässliche Vorstellungen von nicht vorhandenen, vergangenen oder zukünftigen Ereignissen, Personen oder Situationen aufzubauen. In einem zweiten Schritt geht es dann darum, diese Vorstellungen in Symbole, d.h. in Wörter und Sätze zu übersetzen. Vor allem der zweite Schritt ist komplex. Vorstellungen sind allgemein eher ganzheitlich, während das Symbolsystem Sprache streng linear ist: Wörter bestehen aus einer Reihe von Lauten. Sätze bestehen aus einer Reihe von Wörtern. Erzählungen oder Texte bestehen aus einer Reihe von Sätzen. Das heißt: man muss es auf die Reihe bringen! Die Prozesse, welche zu dieser Fähigkeit führen, geschehen in 
der Auseinandersetzung mit der Gegenstandswelt (Ich - Gegenstand), im Besonderen im Symbolspiel. Tatsächlich erfordert das Symbolspiel ganz ähnliche Fähigkeiten wie die Entwicklung der Sprache. Um Erlebnisse in Form symbolischer Handlungen darzustellen, braucht es in einem ersten Schritt ebenfalls die Vorstellung und in einem zweiten Schritt die Fähigkeit, diese in eine lineare Struktur zu übersetzen. Der Unterschied besteht darin, dass die Strukturen im Spiel weniger streng sind als diejenigen der Sprache [2].

\section{Dreiecks-Verbindung}

Letztlich liegt das Besondere der sprachlichen Kommunikation darin, dass es sich immer um eine Verknüpfung der Personenund Gegenstandswelt, also um eine Dreiecks-Verbindung von Ich, Du und Gegenstand handelt. Eine solche Verbindung gelingt dem Kind Ende des ersten Lebensjahres, wenn es nach einem kleinen Erlebnis mit dem Gegenstand erstmals zum Erwachsenen schaut mit dem fragenden Blick „was sagst du dazu?“ bzw. „sagst (oder machst) du's wieder?“. Dieser Blick wird als trian- gulärer oder referentieller Blick bezeichnet und bildet die Basis für die Entwicklung des Sprachverstehens $[3,4]$.

Individuations- und symbolische Prozesse sind die Stützpfeiler der sprachlichen Entwicklung. Sie werden durch den triangulären Blick und das Sprachverstehen verbunden und gemeinsam bilden sie die Grundlage für die Entdeckung der Sprache ( $\bullet$ Abb. 1).

\section{Dynamik früher Spracherwerbsstörungen}

\section{Dezentrierung}

Der wichtigste Schritt für die Entdeckung der Sprache liegt in der Fähigkeit zur Dezentrierung mit der Möglichkeit, das Handlungsresultat zu beachten und diesem eine Bedeutung zu geben. Dieser Schritt ist störungsanfällig, braucht es doch dazu gute Kompetenzen in der Durchführung einer Tätigkeit (Motorik, Praxie, Wahrnehmung) ebenso wie eine stabile Ausgangslage

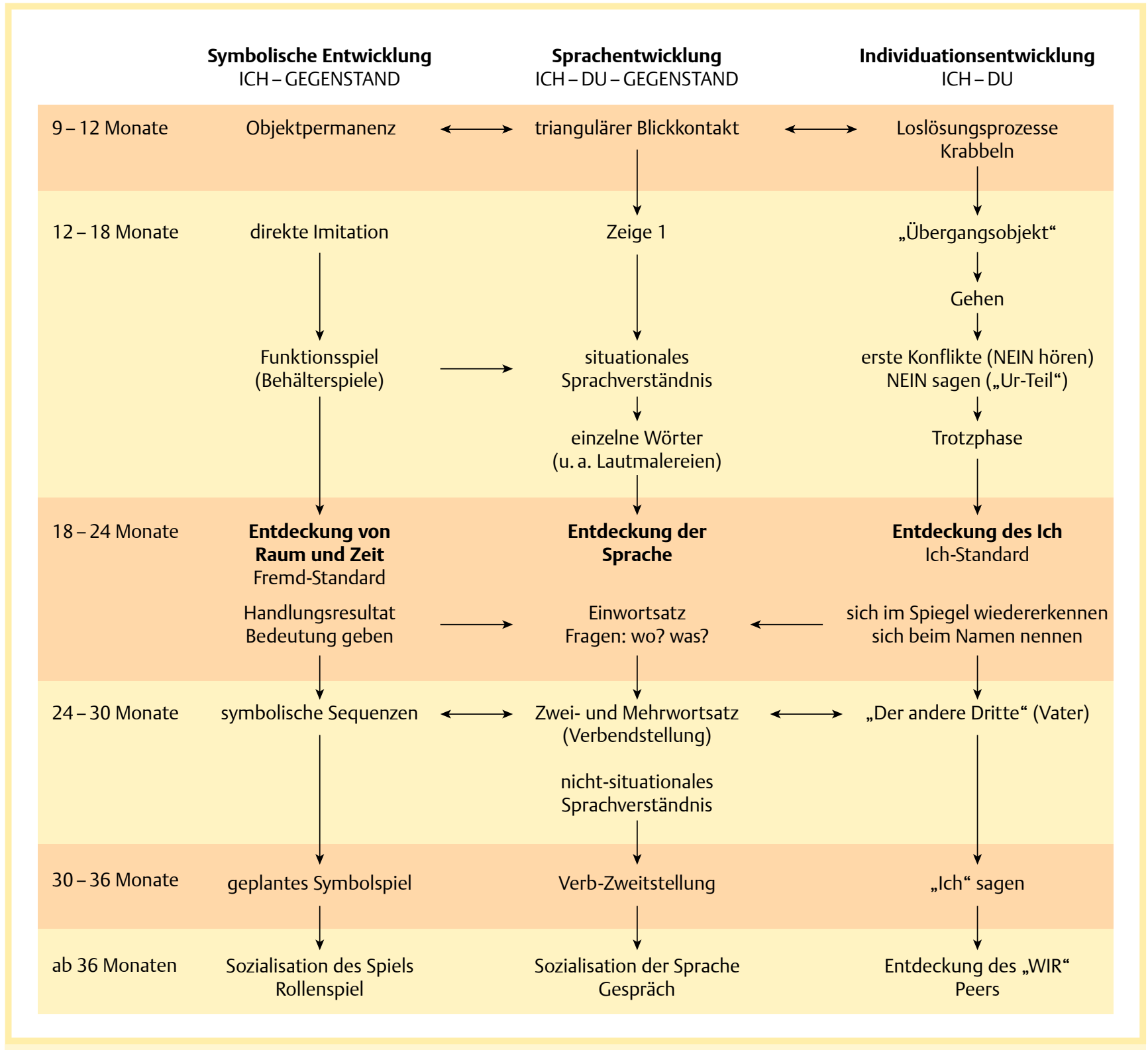

Abb. 1 Schema (Die Entdeckung der Sprache). 
auf der psychologischen Ebene (gut bei sich sein ohne Sorge, die Bezugsperson (aus den Augen) zu verlieren). Unsere Untersuchungen zeigen, dass Kinder, welche Probleme im (motorisch-) praktisch-gnostischen Bereich und/oder in der Loslösung haben, meist im Funktionsspiel stecken bleiben $[5,6]$ und damit Schwierigkeiten haben, verlässliche Vorstellungen aufzubauen.

\section{Bedeutung für den Spracherwerb}

Für den Spracherwerb bedeutet dies vorerst, dass diese Kinder nicht entdecken, was sie mit ihren Wörtern bewirken. Gleichzeitig bleibt das Verstehen der Wörter und später auch der Sätze und Geschichten an die Situation gebunden oder wenig verlässlich. Die Entdeckung und später auch der Aufbau der Sprache verzögern sich, und wenn das Kind zu sprechen beginnt, fehlt das Monitoring, d.h. das Überwachen und Kontrollieren der Verstehens- und Produktionsprozesse [7,8].

\section{Die entwicklungspsychologische Sprachtherapie}

Aufgrund der oben genannten Dynamik liegen die Ziele der logopädischen Arbeit im Frühbereich primär darin, die Individuationsprozesse zu stützen, den Übergang vom Funktionsspiel ins Symbolspiel zu realisieren und das Kind zum Triangulieren und damit zum Sprachverstehen zu führen.

\section{Konzentration auf das Handlungsresultat}

Um dies zu erreichen, gibt es vorerst eine relativ einfache Technik. Die Therapeutin geht immer von dem aus, was das Kind spontan tut. Sie lenkt die Konzentration auf das Handlungsresultat, indem sie seinem Tun durch ihre eigene Handlung oder ihren Kommentar eine Bedeutung gibt [9]. Z. B. drückt das Kind in rein funktionaler Weise auf die Tasten eines Telefons. Die Therapeutin gibt Bedeutung indem sie selbst ein Telefon nimmt, den Hörer abhebt und „hallo?“ sagt, nachfragt und „frustriert“ wieder auflegt, wenn keine Antwort kam. Macht diese Tätigkeit für das Kind Sinn, wird es wieder auf die Tasten drücken - diesmal aber verknüpft mit dem Blick „machst (sagst) du’s wieder?“.

Die Technik des Bedeutung Gebens beruht auf folgenden 3 Prinzipien:

1. Man kann nicht nicht handeln

2. Man kann jeder Handlung eine Bedeutung und damit einen Namen geben

3. Wenn's passt, schaut das Kind, „machst du's wieder?“. Wenn's nicht passt, passiert nichts.

\section{Spiegeln}

Die grundlegende Form von Bedeutung geben ist das Spiegeln, das Wiederholen der Tätigkeit des Kindes. Allein dadurch, dass ein Tun gespiegelt wird, wird es zu „etwas“, das geteilt werden kann. Ebenso grundlegend ist das Kommentieren der Tätigkeit in einer Form, die Absicht unterstellt. Wenn ein Kind bspw. das Feuerwehrauto aus dem Regal holt, kann man dies kommentieren „ah, da kommt die Feuerwehr!“. Oder wenn es eine Kuh auf die Garage stellt, genügt es möglicherweise zu sagen „ah, die Kuh ist auf der Garage - das ist aber spannend!“.

Dieses therapeutische Vorgehen wirkt auf 3 Ebenen:

1. Das Kind erlebt, dass es etwas kann. Damit erfährt es sich als selbstwirksam. Selbstwirksamkeit ist ein zentraler Aspekt der Individuationsentwicklung [10] und einer der wichtigsten Resilienzfaktoren [11],
2. Das Kind erlebt, dass seine Handlungen etwas bewirken. Dieses Erleben bildet die Grundlage für die Dezentrierung und damit für die Entwicklung des Symbolspiels, der Theory of Mind und der Mentalisierungsfähigkeit [12,13].

3. Der fragende Blick „machst du's nochmals?“ trianguliert Personen- und Gegenstandswelt und bildet somit auch Grundlage für die Entwicklung des Sprachverstehens.

\section{Alternative Themenfindung}

Meist gelingt es mit der oben beschriebenen Technik in relativ kurzer Zeit eine oder mehrere Tätigkeiten zu finden, welche für das Kind interessant und damit Ausgangspunkt einer weiteren Entwicklung sind. Es gibt aber auch immer wieder Kinder, welche sich aus unterschiedlichsten Gründen nicht wirklich auf ein gemeinsames Spiel einlassen können. In diesen Situationen kann es unterstützend sein, sich mit der Frage auseinanderzusetzen, welche Themen für dieses Kind interessant sein könnten. So gibt es verschiedene Spiele, welche relativ kulturunabhängig sind, und von fast allen Kindern in bestimmten Entwicklungsphasen gespielt werden. Seewald [14] geht davon aus, dass es sich bei diesen Spielthemen um eine Reaktion auf prägende Ereignisse in der Entwicklung handelt; er bezeichnet sie deshalb als „symbolisches Echo“. Solche Spielinhalte sind beispielsweise das Schneiden, Kleben und Binden als Echo auf die Loslösungsund Individuationsprozesse oder die Spiele mit Kugelbahn, Behältern oder Röhren als Echo auf die Erfahrungen mit dem Aufnehmen, Behalten und Ausscheiden der Nahrung.

\section{Fazit}

„Spiel gilt seit Jahrzehnten als Königsweg für das frühe Lernen.“ [15]. Spielen bildet den Mittelpunkt früher Eltern-Kindund Kind-Kind-Interaktionen. Das Symbolspiel im Besonderen hat eine zentrale Bedeutung für die gesamte kindliche Entwicklung [16].

Speziell im Frühbereich sind wir aber häufig mit dem Problem konfrontiert, dass die Kinder nicht spielen können. Sie sind ungeduldig, unkonzentriert, aggressiv oder zerstörerisch [17]. Sie treffen immer wieder auf Schwierigkeiten, brechen die Tätigkeit ab und sind auf Hilfe und Strukturierung des Erwachsenen angewiesen. Oder sie sind voller Angst, die Bezugsperson (aus den Augen) zu verlieren, kleben an ihr oder müssen immer wieder ihre Anwesenheit kontrollieren.

Wir spielen mit den Kindern, weil sie nicht spielen können. Spielen ist sprachspezifische Therapie, weil es darum geht, Vorstellungen (Repräsentationen) aufzubauen und diese in ein symbolisches System übersetzen zu lernen (18).

Für das gesunde Kind bildet das Spiel eine der wichtigsten Möglichkeiten, sich mit den zentralen Themen bzw. Problemen in seinem Leben auseinanderzusetzen - z.B., wenn ein neues Geschwister auf die Welt kommt, wenn der Nachbarsjunge schon ohne Stützräder fahren kann, wenn die Oma gestorben ist. Kinder mit (sprachlichen) Entwicklungsauffälligkeiten sind meist mit noch viel mehr Schwierigkeiten konfrontiert. Wenn sie nicht spielen können, fehlt ihnen aber die Möglichkeit, sich mit ihren Sorgen, Fragen, Problemen und Unsicherheiten auseinanderzusetzen und so die „heilenden Kräfte im kindlichen Spiel“ zu erfahren.

Spielen lernen bildet deshalb nicht nur eine wichtige Grundlage für den Spracherwerb, sondern für das Leben überhaupt. 


\section{Zur Person}

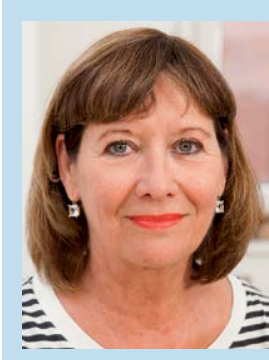

Dr. phil.Barbara Zollinger, dipl. Logopädin. Seit 1983 Lehrbeauftragte an verschiedenen Universitäten und Fachhochschulen. 1994 Gründung des Zentrums für kleine Kinder in Winterthur mit Logopädischer Praxis, Forschungs- und Fortbildungsbereich. 2006 Gründung der Forschungsgruppe $\mathrm{CH}$. Logopädie im Frühbereich. 2007 Auszeichnung der Dr. Hedwig-Stauffer-Stiftung, Autorin und Herausgeberin zahlreicher Fachbücher im Bereich früher Spracherwerbsstörungen.

Interessenkonflikt: Die Autorin gibt an, dass kein Interessenkonflikt besteht.

\section{Literatur}

1 Zollinger B. Spracherwerbsstörungen. Grundlagen zur Früherfassung und Frühtherapie. Bern: Haupt 2008

2 Zollinger B. Die Entdeckung der Sprache. Bern: Haupt 2010

3 Bruner J. Wie das Kind sprechen lernt. Bern: Huber 2002

4 Tomasello $M$. Die Ursprünge der menschlichen Kommunikation. Frankfurt a.M.: Suhrkamp 2009

5 Bürki $D$. Kleine Kinder, die nicht sprechen: eine heterogene Gruppe. In: Zollinger B, Hrsg. Frühe Spracherwerbsstörungen. Kleine Kinder verstehen und Eltern begleiten. Bern: Haupt 2014; 13-42

6 Bürki D, Mathieu S, Sassenroth-Aebischer S et al. Erfassung und Therapie früher Spracherwerbsstörungen. Resultate aus den Dokumentations- und Therapiestudien. Logos Interdisziplinär 2011; 19: 244-250
7 Zollinger B. Die Entdeckung der Sprache. Entwicklungsprozesse, Störungen, Untersuchung, Beurteilung. Pädiatrie up2date 2010; 3: 279-294

8 Zimmermann A. Die Bedeutung des Sprachverstehens und seines Monitorings für die Abklärung. In: Zollinger B, Hrsg. Frühe Spracherwerbsstörungen. Kleine Kinder verstehen und Eltern begleiten. Bern: Haupt 2014; 93-130

9 Zollinger B. Kindersprachen. Kinderspiele. Erkenntnisse aus der Therapie mit kleinen Kindern. Bern: Haupt 2004

10 Dürmüller $C$. „Und jetzt will ich sprechen lernen!“. Die Bedeutung des Selbstkonzepts für die Sprachentwicklung. In: Zollinger B, Hrsg. Frühe Spracherwerbsstörungen. Kleine Kinder verstehen und Eltern begleiten. Bern: Haupt 2014; 179-200

11 Wustmann C. Resilienz. Widerstandsfähigkeit von Kindern in Tageseinrichtungen fördern. Weinheim: Beltz 2004

12 Fonagy P, Gergely G, Jurist EL et al. Affektregulierung, Mentalisierung und die Entwicklung des Selbst. Stuttgart: Klett-Cotta 2004

13 Zollinger $B$. Triadische Beziehungen und Spracherwerbsstörungen: ein neues Konzept für die Früherfassung. In: Zollinger B, Hrsg. Frühe Spracherwerbsstörungen. Kleine Kinder verstehen und Eltern begleiten. Bern: Haupt 2014; 71-92

14 Seewald J. Der verstehende Ansatz in Psychomotorik und Motologie. München: Reinhardt 2007

15 Hauser B. Spiel als notwendige Bedingung gelingender früher Sprachentwicklung. SAL-Bulletin 2014; 151: 5

16 Lillard AS, Lerner MD, Hopkins EJ et al. The Impact of Pretend Play on children's development: A review of the evidence. Psychological Bulletin 2013; 139: 1-34

17 Sassenroth-Aebischer S. Fressen und Gefressenwerden - Zerstören und Wiederaufbauen: Aggressive Spiele in der Sprachtherapie. In: Zollinger B, Hrsg. Frühe Spracherwerbsstörungen. Kleine Kinder verstehen und Eltern begleiten. Bern: Haupt 2014; 153-178

18 Zollinger B. Und wenn sie nicht spielen können? SAL-Bulletin 2014; 153: $5-16$ 